\title{
SOUAMOUS CELL CARCINOMA DEVELOPED IN CHRONIC OSTEOMYELITIS
}

doi: 10.2478/rojost-2018-0077

\author{
M.I. Zaharia ${ }^{1}$, A.M. Oproiu'1,2, M. Costache ${ }^{1,2}$, D. Popescu ${ }^{1}$, N. Dumitrescu ${ }^{1}$, A. Varga ${ }^{3}$ \\ ${ }^{1}$ University Emergency Hospital, Bucharest, Romania \\ 2"Carol Davila" University of Medicine and Pharmacy, Bucharest, Romania \\ ${ }^{3}$ County Emergency Hospital, Buzău, Romania
}

Introduction. Squamous cell carcinoma is the second as frequency in malignant tumors of the skin, representing $20 \%$ of this type of cancer. Inferior lip squamous carcinoma is most frequent, of $94 \%$, because the trauma, sunburns, smoke and radiations are more frequent. Metastasis at distance in the area with chronic osteomyelitis is a rare situation.

Material and method. A 78-year-old male, treated for inferior lip carcinoma, without any oncological treatment, was admitted in our clinic for a leg tumor on the anterior part, developed over a scar after osteomyelitis treated in the adolescence. The tumor growth was quick. We decided the treatment plan from the point of view of a multidisciplinary approach, plastic surgeon-orthopedistoncologist-dermatologist. A biopsy from the extraosseous tumor was performed and after the histological confirmation, the resection of the tumor block was made. For the confirmation of the bone invasion, we had to repeat the immunohistochemistry twice because the first result was negative. Until the final decision, the patient had an external fixation on the leg. A large tissue appeared on the area of resection, the aspect being suggestive for granulation. Amputation was decided after the complementary investigations and another biopsy from the granulation tissue. Results and conclusions. Squamous cell carcinoma of the lip needs prophylactic laterocervical ganglia resection for limiting metastasis.

Keywords: squamous cell carcinoma, chronic osteomyelitis, metastasis 